\title{
Exercise-based Cardiovascular Rehabilitation in Chronic Heart Failure: does Exercise Intensity Matter?
}

\author{
Gabriel Dias Rodrigues ${ }^{\circ}$ \\ Universidade Federal Fluminense - Physiology and Pharmacology, Niterói, RJ - Brazil \\ Editorial referring to the article: Continuous Aerobic Training and High Intensity Interval Training Increase Exercise Tolerance in Heart \\ Failure Patients: A Retrospective Study
}

It is well recognized that chronic heart failure (CHF) is a multifactorial syndrome that reduces physical exercise tolerance. ${ }^{1}$ Exercise-based cardiac rehabilitation program is on the frontline of non-pharmacological therapies for CHF patients, counterbalancing the disease-induced issues of physical capacity. ${ }^{2}$

Since a successful exercise rehabilitation program should be efficient and safe, ${ }^{2}$ the search for optimal combinations of exercise volume and intensity has sparked interest in the current literature. Although studies have shown feasible combinations of low volume and high intensity, and vice versa, the optimal dose-response of exercise in cardiovascular rehabilitation remains unknown. Classically, aerobic training with moderate intensity (i.e., between $60 \%$ and $80 \% \mathrm{VO}_{2 \text { peak }}$ or heart rate reserve - HRreserve) is a safe approach to improve physical capacity and provide a cardioprotective effect in CHF disease. ${ }^{3}$ As a promising method, short bouts of high-intensity exercise, called high-intensity interval training, seems to be superior than moderate continuous training to reverse left ventricle remodeling and improve aerobic capacity, endothelial function, and quality of life in post-infarction heart failure patients. ${ }^{4}$

\section{Keywords}

Exercise; Heart Diseases; High-Intensity Interval Training; Cardiac Rehabilitation.
In the current issue of the International Journal of Cardiovascular Sciences, Busin and colleagues ${ }^{5}$ report that both high-intensity interval training and mild-intensity continuous training increase exercise tolerance in CHF patients. However, in their study, a greater number of parameters related to exercise tolerance (e.g., treadmill speed, incline, and time) was improved by high-intensity interval training than conventional training. While continuous aerobic training increased the treadmill speed at the first ventilatory threshold, high-intensity interval training increased both the speed and incline of the treadmill for $\mathrm{VO}_{2 \text { peak }}$ and the second ventilatory threshold. On the other hand, neither high-intensity nor moderate training changed $\mathrm{VO}_{2 \text { peak }}$ or post-exercise heart rate recovery. Although the $\mathrm{VO}_{2 \text { peak }}$ has been used for decades as a universal "gold-standard" of exercise tolerance, reflecting the severity of $\mathrm{CHF}$ disease ${ }^{6}{ }^{6}$ alternative markers that may indicate an adaptation from exercise-based rehabilitation in CHF seem necessary.

Therefore, novel combinations of high- and lowintensity exercise with different exercise volumes should be investigated in further studies of cardiovascular rehabilitation in CHF patients. Also, additional markers of physical performance in exercises programs should be considered, since exercise tolerance in CHF seems to be multifactorial. Thus, the question remains open: does exercise training intensity matter?

Mailing Adress: Gabriel Dias Rodrigues

Rua Professor Hernani Pires de Melo, 101. Postal Code: 24210-130, São Domingos, Niterói, RJ - Brazil

E-mail: dias5gabriel@gmail.com

DOI: https://doi.org/10.36660/ijcs.20210128 


\section{References}

1. Mosterd A, Hoes AW. Clinical Epidemiology of Heart Failure. Heart. 2007;93(9):1137-46. doi: 10.1136/hrt.2003.025270.

2. Leon AS, Franklin BA, Costa F, Balady GJ, Berra KA, Stewart KJ, et al. Cardiac Rehabilitation and Secondary Prevention of Coronary Heart Disease: An American Heart Association Scientific Statement from the Council on Clinical Cardiology (Subcommittee on Exercise, Cardiac Rehabilitation, and Prevention) and the Council on Nutrition, Physical Activity, and Metabolism (Subcommittee on Physical Activity), in Collaboration with the American association of Cardiovascular and Pulmonary Rehabilitation. Circulation. 2005;111(3):369-76. doi: 10.1161/01.CIR.0000151788.08740.5C.

3. Piepoli M, Maugeri F, Campana M, Ferrari R, Giordano A, Scalvini S et al. Experience from Controlled Trials of Physical Training in Chronic Heart Failure. Protocol and Patient Factors in Effectiveness in the Improvement in Exercise Tolerance. European Heart Failure Training Group. Eur Heart J. 1998;19(3):466-75. doi: 10.1053/euhj.1997.0736.
4. Wisløff U, Støylen A, Loennechen JP, Bruvold M, Rognmo Ø, Haram PM, et al. Superior Cardiovascular Effect of Aerobic Interval Training Versus Moderate Continuous Training in Heart Failure Patients: A Randomized Study. Circulation. 2007;115(24):3086-94. doi: 10.1161/ CIRCULATIONAHA.106.675041.

5. Busin D, Lehnen AM, Tairova OS, Comparsi EP, Carneiro D, Potter M, Deresz LF, et al. Continuous Aerobic Training and High Intensity Interval Training Increase Exercise Tolerance in Heart Failure Patients: A Retrospective Study. Int J Cardiovasc Sci. 2021; 34(5):508-514. doi: 10.36660/ijcs.20200124.

6. Guazzi M, Adams V, Conraads V, Halle M, Mezzani A, Vanhees L, et al. EACPR/AHA Scientific Statement. Clinical Recommendations for Cardiopulmonary Exercise Testing Data Assessment in Specific Patient Populations. Circulation. 2012;126(18):2261-74. doi: 10.1161/ CIR.0b013e31826fb946. 